\section{Two Dimensions and Two States in DNA Nanotechnology}

\author{
http://www.adeninepress.com/jbsd
}

... a flock of crazy prophets, that by staring at a crystal can fill it with more fancies than there are herrings in the sea.

\begin{abstract}
The construction of periodic matter and nanomechanical devices are central goals of DNA nanotechnology. The minimal requirements for components of designed crystals are [1] programmable interactions, [2] predictable local intermolecular structures and [3] rigidity. The sticky-ended association of DNA molecules fulfills the first two criteria, because it is specific and diverse, and it results in the formation of B-DNA. Stable branched DNA molecules permit the formation of networks, but individual single branches are too flexible. Antiparallel DNA double crossover (DX) molecules can provide the necessary rigidity, so we use these components to tile the plane. It is possible to include DNA hairpins that act as topographic labels for this 2-D crystalline array, because they protrude from its plane. By altering sticky ends, it is possible to change the topographic features formed by these hairpins, and to detect these changes by means of AFM. We can modify arrays by restricting hairpins or by adding them to sticking ends protruding from the array.
\end{abstract}

Although individual branched junctions are unsuitable for use as crystalline components, parallelograms of four 4-arm junction molecules are sufficiently rigid that they can be used to produce $2 \mathrm{D}$ arrays. The arrays contain cavities whose dimensions are readily tuned by changing the edges of their parallelogram components. We have used these arrays to measure directly the angle between the helices of the Holliday junction.

The rigidity of the DX motif can also be exploited to produce a nanomechanical device predicated on the B-Z transition. Two DNA double crossover molecules have been joined by a segment of DNA capable of undergoing the B-Z transition. In the B-conformation, the unconnected helices of the two molecules are on the same side of the connecting helix, whereas in the $\mathrm{Z}$ conformation they are on opposite sides, leading to movements of as much as $60 \AA$. This effect is shown by fluorescence resonance energy transfer, because dyes attached to the unconnected helices have different separations in the two states.

\section{DNA and Molecular Design}

DNA is well-known as the genetic material of living systems. Knowledge of its double helical structure (1) has had an enormous impact on our understanding of life's processes. The double helix may well come to symbolize our civilization to future civilizations, in much the same way that the pyramids of Egypt, the ruins of Machu Picchu, the cathedrals of Medieval Europe, the Great Wall of China, the Parthenon and the Taj Mahal are structural emblems to us of the societies that built them. However, in contrast to these cultural icons, which represent the apotheoses of their respective civilizations, the DNA double helix represents the first step in the development of molecular biology. In the years since the proposal of its structure, the fundamentals of molecular genetics and gene expression have been worked out, the biotechnology industry has developed, diagnostics and criminology have been

\author{
Nadrian C. Seeman ${ }^{1 *}$, \\ Furong Liu', \\ Chengde Mao', \\ Xiaoping Yang1, \\ Lisa A. Wenzler ${ }^{1}$, \\ Ruojie Sha1, \\ Weiqiong Sun', \\ Zhiyong Shen1, \\ Xiaojun Li', \\ Jing Qi', \\ Yuwen Zhang1, \\ Tsu-Ju Fu', \\ Junghuei Chen 1 \\ and Erik Winfree ${ }^{2}$ \\ ${ }^{1}$ Department of Chemistry, \\ New York University, \\ New York, NY 10003 \\ ${ }^{2}$ Computation and Neural Science, \\ California Institute of Technology \\ Pasadena, CA 91125
}

*Phone: 212-998-8395;

Fax: 212-260-7905;

E-mail: ned.seeman@nyu.edu 
revolutionized, and the sequences of several simple genomes, as well as a portion of the human genome, have been determined.

A central reason for the rapidity of these advances is the simplicity of the complementary pairing rules that determine DNA structure. It is a complicated and chancy enterprise to estimate in three dimensions how two protein molecules will associate with each other. DNA is an entirely different matter. Knowing that under standard conditions adenine (A) pairs with thymine $(\mathrm{T})$ and that guanine $(\mathrm{G})$ pairs with cytosine (C) allows everyone to predict the complement to any DNA sequence, with a minimum of effort. When put in a chemical context, this is remarkable: Inter-molecular associations can be devised and programmed with both high specificity and large diversity. In addition, current synthetic methods enable the routine synthesis of oligodeoxynucleotides containing over 100 residues (2). These facts have led to two new areas, DNA nanotechnology (35), and DNA-based computation (6). The goals of DNA-based computation are to combine the enormous parallelism of molecular computation with the inherent specificity of DNA recognition to devise and execute algorithms that can solve problems intractable to current computational approaches (6). The goals of DNA nanotechnology are the construction of DNA objects, devices and periodic arrays based on the specificity of DNA interactions; key among the goals of DNA nanotechnology is the use of the architectural features of DNA to direct the arrangement of heteromolecules into particular positions and associations (3-5), and to extend these structures to the macroscopic scale. There are implications of DNA nanotechnology for DNAbased computation (7), but we will focus on the structural aspects of DNA nanotechnology here, and review recent progress in array formation and device construction. Earlier work in this field was dependent almost exclusively on indirect evidence obtained by gel electrophoretic analyses; the work described here on array formation relies on direct evidence from atomic force microscopy (AFM).

DNA Nanotechnology. The biotechnology revolution is based on the ability to combine DNA molecules by means of complementary sticky ends (8). Sticky ended association, by ligation or hydrogen bonded cohesion, takes place between single-stranded overhangs on the ends of DNA duplexes. Although it uses the same hydrogen-bonded interactions that hold individual double helices together, sticky ended association accomplishes something different: It brings two different double helices together. Thus, the sticky ends act as tags to target the intermolecular associations of two DNA molecules.

The DNA double helix is topologically linear, in the sense that it is not branched. Thus, sticky ended association of duplex molecules can lead only to longer (or circular) DNA molecules. However, synthetic DNA need not be only linear. It is possible to design branched DNA molecules, termed 'junctions', with sticky ends (3), and to direct their associations with the same specificity and diversity that the associations of linear molecules can be directed. An illustration of this concept is shown in Figure 1, where four 4-arm branched junctions are seen associ-

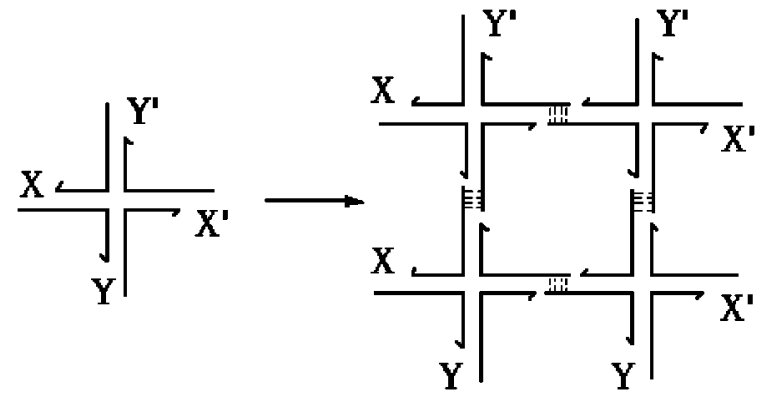

Figure 1: Formation of a Two-Dimensional Lattice from an Immobile Junction with Sticky Ends. $\mathrm{X}$ is a sticky end and $\mathrm{X}^{\prime}$ is its complement. The same relationship exists between $\mathrm{Y}$ and $\mathrm{Y}^{\prime}$. Four of the monomeric junctions on the left are complexed in parallel orientation to yield the structure on the right. Note that $\mathrm{X}$ and $\mathrm{Y}$ are different from each other, as indicated by the pairing in the complex. DNA ligase can close the gaps left in the complex. Note, also, that the complex has maintained open valences, so that it could be extended by the addition of more monomers.

ating to form a quadrilateral. Thus, it is possible to imagine preparing species that form multiply-connected stick figure objects and networks. A network is said to be N-connected if each of its vertices is connected to $\mathrm{N}$ other vertices by edges of the network (e.g., 9,10). In DNA nanotechnology, the vertices are the branch points of junctions, and the edges are double helices that connect them. Figure 1 shows that edges are formed by sticky ended cohesion between the arms of two different junctions. Junctions with three (11), four (12), five and six (13) arms have been characterized.

Three-arm junctions are known to be flexible (11), and 4-arm junctions are known to arrange their helices in two stacking domains (14); however, they have also been shown to exhibit flexibility $(15,16)$. If one is dealing with flexible components, it is clear that the control over the products is only at the topological level, and not at the geometrical level. Likewise, characterization of products can be done only at the topological level. Geometrical control requires rigid components. We will describe below that it is now possible to produce rigid components from branched DNA molecules.

Construction of Individual Molecules. The earliest multiplyconnected molecules constructed by means DNA nanotechnology were stick polyhedra. These were molecules with the connectivities of a cube (17) and of a truncated octahedron (18). These molecules are illustrated in Figure 2, which shows the basic topological arrangements of the molecules, on the left, and the edges replaced with DNA double helices, on the right. These molecules were assembled by ligation, and it was necessary to separate completely ligated molecules from failure products by electrophoresis. The yields of these molecules were very low, and it was clear that they were inappropriate species to use as starting components to produce macroscopic or even mesoscopic materials.

As noted above, we cannot demonstrate the shapes of these molecules, nor do we know even whether they have unique shapes; for example, the 'cube' could be shaped like a rhombohedron, or any other parallelepiped. The helicity of the double helix leads 

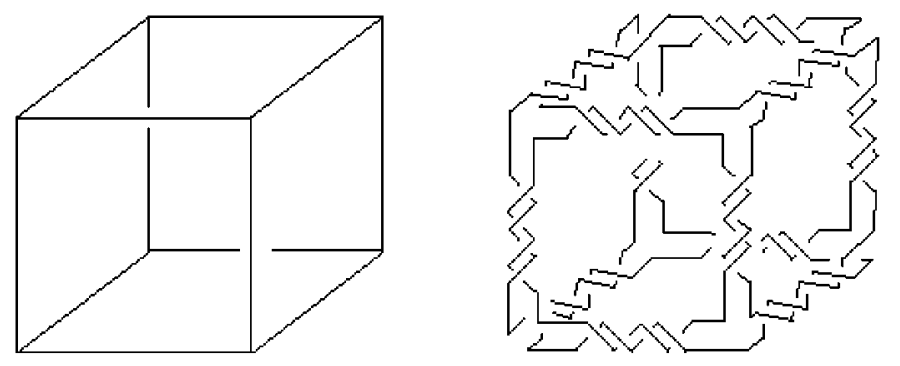

Periodic Matter. The deliberate construction of periodic matter is a key goal of DNA nanotechnology. The conventional crystallization of macromolecules is often a very difficult procedure, which is highly sensitive to subtle aspects of the crystallization conditions (e.g., 21). In principle, one could utilize an alternative, consisting of DNA cages that contain ordered guests (3). The cages themselves could be directed to associate by means of sticky-ended associations. A sample cage with oriented guests is illustrated in Figure 3a. The conditions in which sticky ends cohere are well-known and easy to control. The replacement by sticky ends of the unknown and sensitive contacts that currently
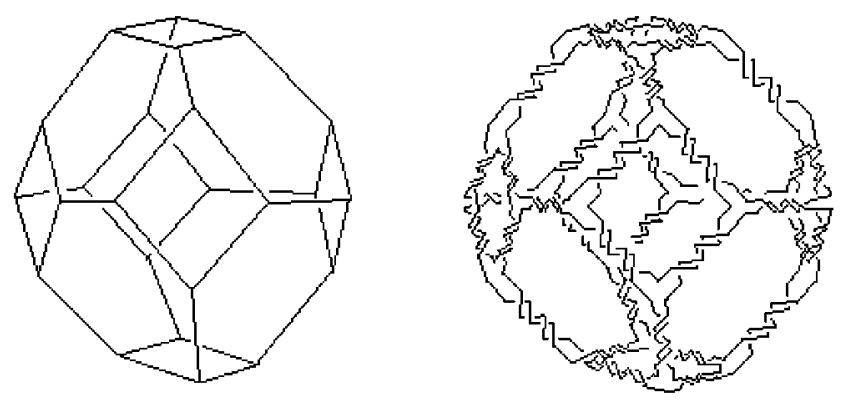
stabilize macromolecular crystals would offer a robust method for producing periodic matter on the nanometer scale. In addition to organizing the arrangement of biological macromolecules for structural analysis, it should be possible to use this system to organize molecular electronic components (22); success in this venture would help to decrease the size of computer components. An example of branched junctions organizing molecular electronic components pendent from DNA is shown in Figure $3 \mathrm{~b}$.

Figure 2: Stick Polyhedra Constructed from DNA, a Cube and a Truncated Octahedron. The drawings on the left show the graphs of an ideal cube (above) and an ideal truncated octahedron (below). The drawings on the right illustrate that each edge of the figure has been transformed to two turns of double helical DNA. The twisting is confined to the central portion of each edge for clarity, but in actuality it extends from vertex to vertex. There are two turns per edge in each figure, so each face corresponds to a single strand. Both molecules are drawn as though they were constructed from 3-arm junctions, but the truncated octahedron has been constructed from 4-arm junctions. Thus, there is another arm on the square strands at each vertex, but this arm has been omitted for clarity.

to the linking of the strands that make up each edge (19). When there is an integral number, $\mathrm{L}$, of double helical turns in an edge, each face of the molecule corresponds to a cyclic single strand, linked L times to each of the neighbors with whom it shares an edge. Both the cube and the truncated octahedron contain two turns per edge. Hence, the cube is a hexacatenane, and the truncated octahedron is a 14-catenane, in which each circle is linked twice to its neighbors. It is possible to characterize the topology of the molecules by means of denaturing gel electrophoresis, so long as molecular standards can be built (20).

a
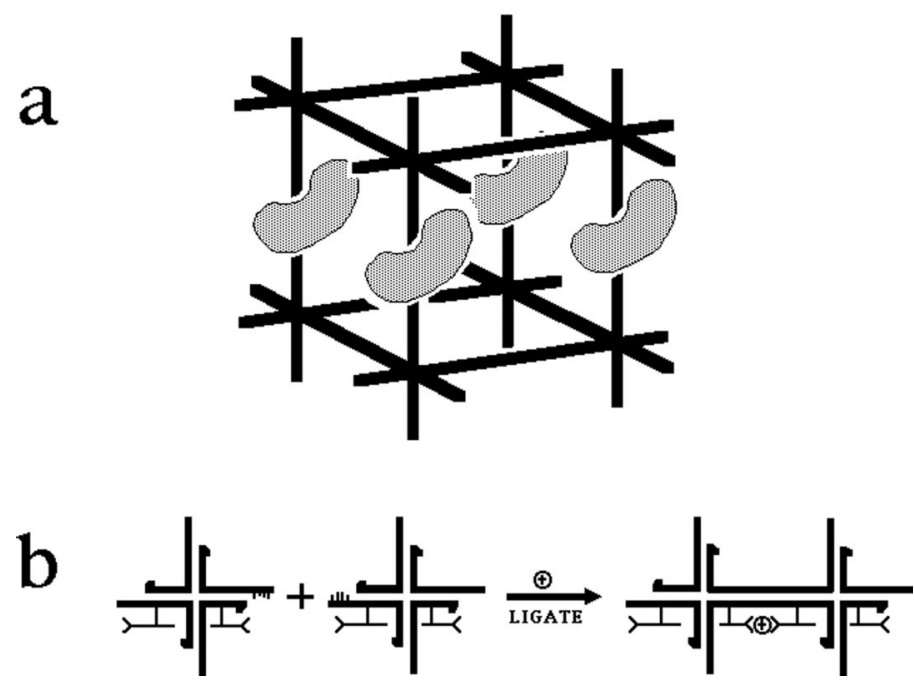

The requirements for lattice components are more stringent than for the assembly of isolated objects, such as the polyhedra described above. The variation of sticky ends can produce particular closed species in small flexible systems, such as the cube and truncated octahedron. From the standpoint of sequence, these are low symmetry structures, because all of the sticky ends can be made unique. However, the construction of periodic matter entails high symmetry components, because the opposite faces of each unit cell are complementary. There are at least three key elements necessary for the control of three-dimensional structure in molecular construction involving high symmetry: [1] The predictable specificity of intermolecular interactions between components; [2] the structural predictability of intermolecular products; and [3] the structural rigidity of the components. The first criterion addresses intermolecular affinity, without regard to structure. We have discussed above that sticky ends are very specific, highly diverse, and readily programmed; thus, DNA branched junctions tailed with sticky ends fulfill this requirement. The second point is somewhat more subtle: The geometry by which two molecules bind is key to engineering the shape of the products in space. For example, enough is known about antibody structure today that one could predict reliably where an antigen would bind on its surface (e.g., 23); however, to know the precise geometry of the interaction would require further physical data, such as a crystal structure. In contrast to this situation, the cohesion of DNA sticky ends yields precise and predictable geometry, because the ligated or hybridized product is double helical B-DNA (24).

Figure 3: Future Applications of DNA Nanotechnology. (a) A Guest in a Simple Cubic Lattice. The DNA lattice is drawn as a portion of a simple cubic lattice made from 6 -arm junctions. The guests are represented by the kidney bean-shaped features in every unit cell. If the guests can be aligned parallel to each other, their structures can be determined by X-ray crystallography. (b) DNA as Scaffolding. Two branched junctions are shown, and a molecular wire is attached to them. When the two junctions cohere with each other, so does the molecular wire, which forms a synapse. This drawing represents one way that DNA could be used to organize molecules whose properties are best suited to a particular molecular task. 
Unfortunately, the flexibility of branched DNA molecules does not fulfill the third criterion. We describe below two different solutions to this problem that enable the construction of periodic matter: The combination of two branched junctions into a double crossover (DX) molecule, and the combination of four branched junctions into a parallelogram.

Nanomechanical Devices. The objects and arrays described above are static species, in that they do not contain moving parts. In addition to such static species, it is desirable to assemble nanomechanical devices from DNA. The simplest device is a rigid object that responds to an external stimulus by changing structure in a predictable fashion. The rigidity is necessary so that the structures before and after the transition can be characterized as different. It is clear that this type of construction requires rigid DNA components, in the same way that periodic matter does. We describe below how DX molecules have been adapted to act as components of a DNA-based nanomechanical device.

\section{Periodic Arrangements of DNA Double Crossover Molecules}

DNA Double Crossover Molecules. DNA double crossover (DX) molecules have been suggested as intermediates in recombination involving double strand breaks $(25,26)$, and they are known to be intermediates in meiosis (27). They consist of two 4-arm branched junctions that are joined at two adjacent double helical arms. When modeled in small systems (28), there are five different motifs, three of which have parallel helix axes, and two have antiparallel helix axes (Figure 4). Both types are further subdivided by whether their crossover points are separated by an even (DPE or DAE) or odd (DPO or DAO) number of double helical half-turns; there are two types of DPO molecules, depending on whether the extra half turn corresponds to a wide (DPOW) or narrow (DPON) groove. It is also possible to make a derivative of DX molecules by including one or more junctions in the motif; Figure 4 shows a molecule in which an extra junction has been added to a DAE molecule, to produce a DAE $+\mathrm{J}$ molecule (29). Although shown as having its helix axis coplanar with the axes of the other two helical domains, this is not necessary. We will describe below motifs in which extra helices protrude from the plane of the DX molecule.

Figure 4: Double Crossover Molecules and Their Ligation Products. The top row contains the three parallel isomers of double crossover (DX) molecules, DPE, DPOW and DPON; 'P' in their name indicates their parallel structure. Arrowheads indicate $3^{\prime}$ ends of strands. Strands drawn with the same thickness are related by the vertical dyad axis indicated in the plane of the paper. DPE contains crossovers separated by an even number (2) of half-turns of DNA, DPOx by an odd number; in DPOW, the extra half turn is a major groove spacing, in DPON, it is a minor groove spacing. The middle row illustrates two other DX isomers, DAE, and DAO. The symmetry axis of DAE is normal to the page (the symmetry is broken by the nick in the central strand); the symmetry axis of DAO is horizontal within the page; in the case of DAO, strands of opposite thickness are related by symmetry. DAE $+\mathrm{J}$, in the second row is a DAE in which an extra junction replaces the nick shown in DAE. Ligation products of the DAE, DAO and DAE+J molecules are shown at the bottom of the drawing, where one domain has been capped by hairpins. Ligation of DAE and DAE+J leads to a reporter strand, but ligation of DAO leads to a polycatenated structure. It is possible to reorient the extra helix of the $\mathrm{DAE}+\mathrm{J}$ motif so that it protrudes from the plane containing the helix axes of the other two domains.
Parallel DX molecules are not well behaved in model systems, even though they are the DX molecules relevant to biological systems, where the crossover separations are usually much larger. Antiparallel molecules are well behaved, and hence they have received all of our attention in this work. We assay rigidity in a ligation-closure experiment; if the molecule produces only linear products [or only a particular cyclic product], then we regard it as sufficiently rigid for purposes of nanoconstruction. The ligation-closure experiment usually relies on the presence of a single strand, called a reporter strand (29), whose fate reflects that of the complex: If it remains linear, so does the complex; if it cyclizes, so does the complex. The bottom of Figure 4 shows that ligating both the DAE molecule and the DAE $+\mathrm{J}$ molecule results in the presence of a reporter strand, drawn more darkly than the other strands. However, the ligation of DAO produces only a catenane, which is harder to analyze. Thus, the assays reported so far have been restricted to DAE-like molecules. Fortunately, these molecules have passed the oligomerization assay successfully (29), suggesting that it is possible to use them to construct periodic DNA arrangements.
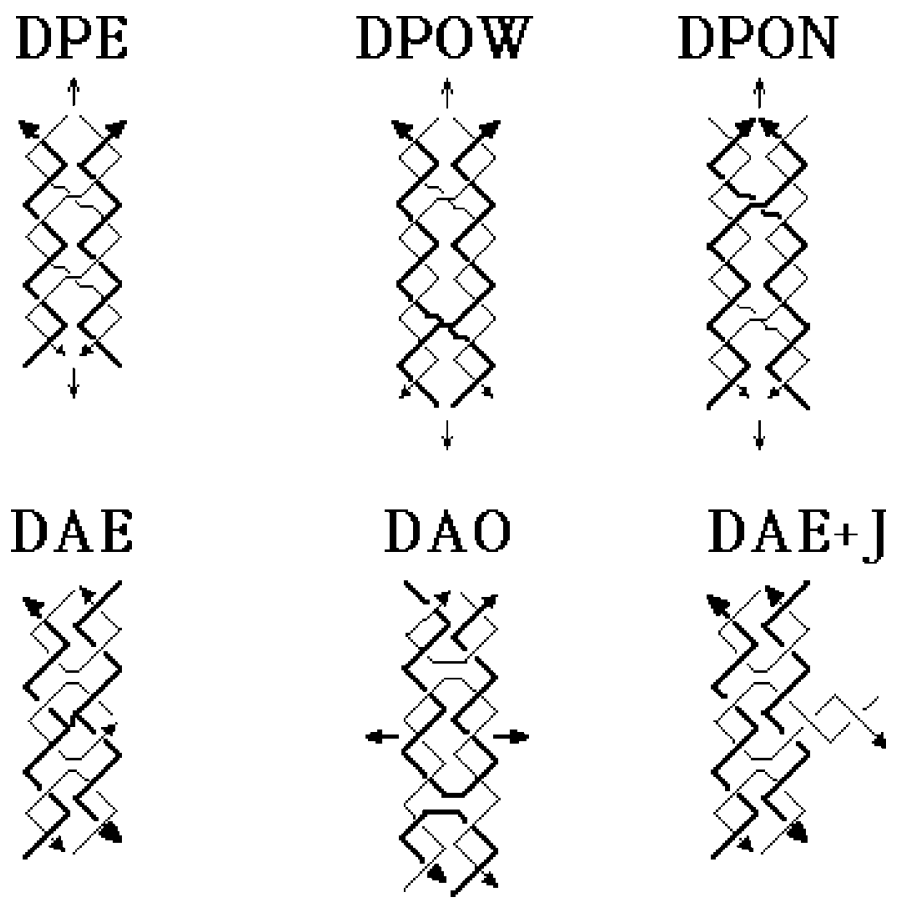

\section{Ligated DAE}
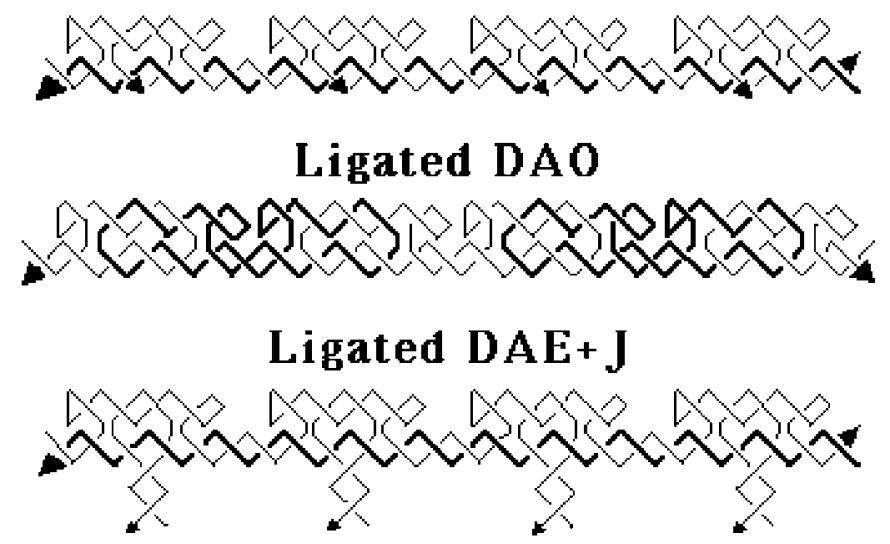
Ligated 1D Arrangements of Triangles with a DX Edge. A simple test of the suitability of DX molecules for the construction of larger arrangements is to ligate them together in one dimension. The experiment described above that demonstrated their apparent rigidity was interpreted on the basis of a denaturing gel electrophoresis of a reporter strand (29). A more direct measurement is to observe the products in the AFM. So as to delimit the individual units, we have made a DNA triangle, one of whose edges consists of a DAE motif. One helical domain forms a side of the triangle, and the other contains a complementary pair of sticky ends that are designed to be ligated together. The molecule is shown on the left of Figure 5a. The separation of its sticky ends is 4.5 turns. When ligated, the triangles alternate their orientations about the central helix. Two vertices on the same side are expected to be separated by 9 double helical turns, or about $32 \mathrm{~nm}$. This is the result seen in the AFM images (30).

All microscopic images must be challenged, because of the possibility of getting the result one desires through artifact. The mesoscopic images produced here are expected to be derived from simple geometrical building blocks on the molecular scale, so it is easy to design tests to challenge them. These tests show the power of directing molecular self-assembly by sticky ends. We have devised two different challenges to the interpretation of this image. The first is shown in Figure 5b, where we have altered the sticky ends on the triangle, and have added a simple DX molecule, four turns long, to the mixture. The expected separation of triangular vertices is now 17 turns, about $60.5 \mathrm{~nm}$. This result is also seen (30). As a final test, we lengthen the simple DX molecule to 4.5 turns, so that the vertices should all be on the same side of the connecting helix, again separated by about $32 \mathrm{~nm}$. This result is also seen, confirming the interpretation of the images (30). The molecules are not as straight as the schematics, but it is possible to fit scaled versions of the schematics directly onto the images (30).

Hydrogen Bonded 2D Arrays from DX Molecules. Figure 6 illustrates the way in which DX molecules can be used to construct two dimensional periodic arrays. Figure 6a illustrates two DX molecules, $\mathbf{A}$ and $\mathbf{B}^{*}$. A is a conventional DX molecule, and $\mathbf{B} *$ is a $\mathrm{DX}+\mathrm{J}$ or $\mathrm{DX}+2 \mathrm{~J}$ molecule, containing one or two extra hairpins. The helix axes of the hairpins are designed not to lie in the plane of the other helix axes of the DX, as illustrated in Figure 4, but are directed instead out of that plane. In this way, they will protrude from the plane, and will provide topographic markers visible in the AFM. These features are indicated by the filled circle in the middle of the diagram. The sticky ends are represented geometrically, so that complementary surfaces are illustrated. These molecules are not ligated, but only cohere by hydrogen bonding. The molecules are about $16 \mathrm{~nm}$ long, and $4 \mathrm{~nm}$ wide, so the hairpins should provide a striped feature in the pattern every 32 nanometers. This is seen when these molecules are examined by AFM, regardless of whether the DX units are DAE or DAO molecules (31).

A test of the interpretation of the AFM image obtained is illustrated in Figure 6b. This drawing illustrates four different DAE molecules, A, B, C and $\mathbf{D} *$. Only $\mathbf{D}^{*}$ is a $\mathrm{DX}+\mathrm{J}$ motif, so the striped features should now be separated by $64 \mathrm{~nm}$, instead of 32 $\mathrm{nm}$. This result is seen in AFM images of the ABCD* array, confirming that the stripes seen in the $\mathbf{A B} *$ array actually derive from the designed array, and are not merely artifacts (31). As seen with the ligation experiments involving triangles, the diversity and programmability of sticky ends provides a powerful tool for verifying the interpretation of AFM images.

It is worth noting that much less effort has gone into the preparation of the DX components than into the preparation of the ligated polyhedra. The individual DX molecules are merely hybridized together. Consequently, it is easy to prepare large quantities of starting components, without ligating the species together. Likewise, the components are held together by hydrogen bonding, so one is not dependent on ligation to create the arrays. This hydrogen bonding approach appears to be appropriate for future studies aimed at producing macroscopic assemblies.

\section{Substrates}

a

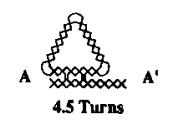

b

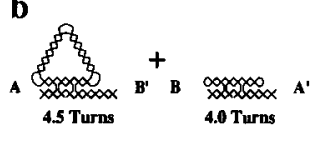

c

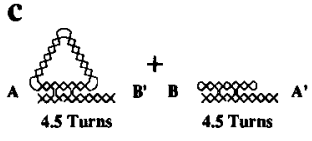

Expected Ligation Products
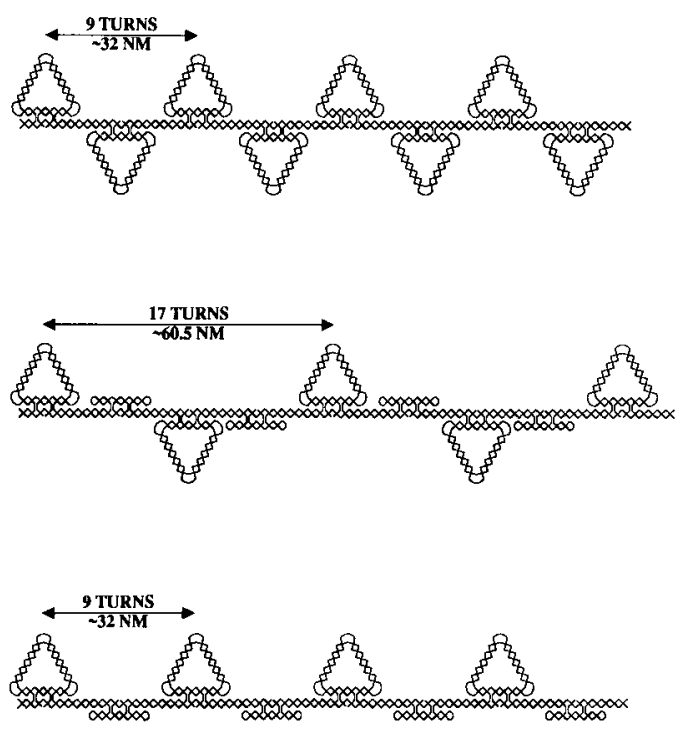

Figure 5: Triangle Ligation Experiments. Three ligation experiments are shown in this diagram. The substrates are shown on the left, and the expected ligation products are shown on the right. The triangle in panel $(a)$ is prepared with complementary sticky ends, and ligated to produce a linear array in which the vertex opposite the DX points alternately in opposite directions, because the length of the ligated helix is 4.5 turns of DNA; successive vertices on the same side of the central helix are expected to be separated by about $32 \mathrm{~nm}$. The experiment in panel (b) illustrates the ligation of the same triangle, but this time alternating with a DX molecule whose ligation domain is 4.0 turns. One domain on the DX molecule is sealed with hairpin loops. The sticky ends on the triangle (represented as A and $\mathrm{B}^{\prime}$ ) and on the DX (represented as B and $A^{\prime}$ ) enforce this strict alternation. This structure is expected to spread out the separation of successive vertices on the same side of the central helix to about $60.5 \mathrm{~nm}$. The experiment in panel (c) is similar to the middle experiment, except that the ligation domain of the DX molecule is 4.5 turns. This is expected to produce a product in which all of the triangles are on the same side of the central helix, and in which the vertices are separated by $32 \mathrm{~nm}$. 

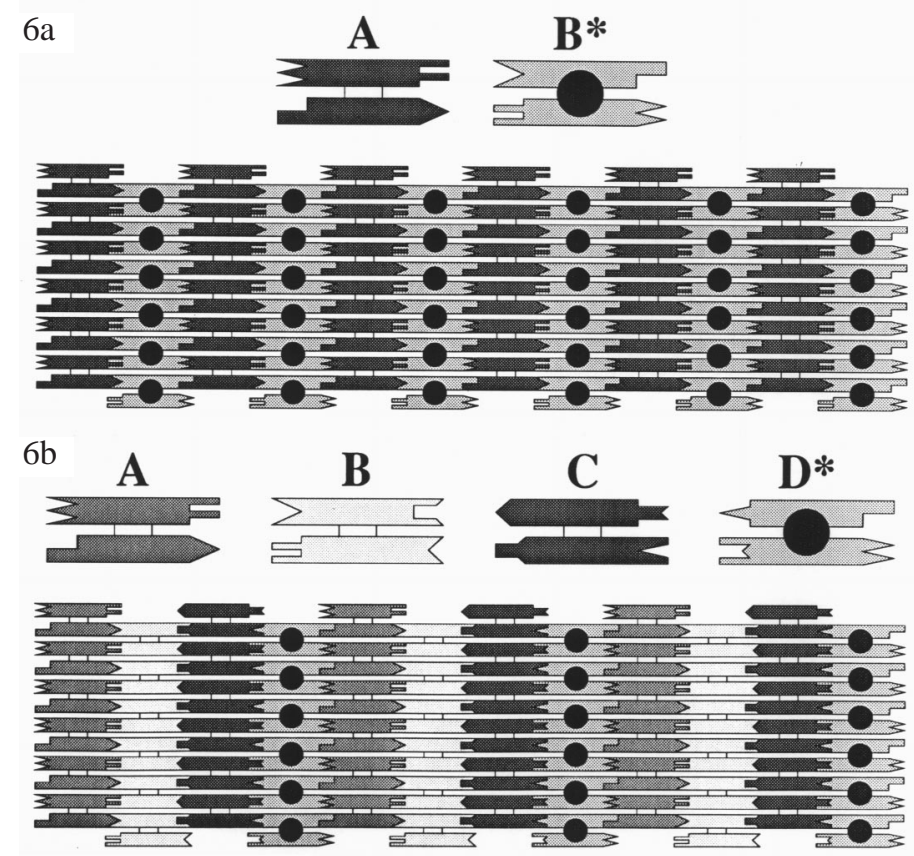

Modification of DX Patterns. It would be economical to produce a multitude of patterns from a basic starting set of components. This could be done if one were able to modify a topographic pattern generated by an array. The topographic features that produce patterns in Figure 6 are derived from DNA. Consequently, it should be possible to modify those patterns by using standard molecular biological operations, such as restriction, ligation or hybridization. Figure 7a illustrates alteration of a pattern by the action of restriction enzymes. The upper portion of the drawing shows four components, $\mathbf{A}, \mathbf{B}^{\#}, \mathbf{C}$ and $\mathbf{D}^{*}$. $\mathbf{B}^{\#}$ and $\mathbf{D}^{*}$ are both $\mathrm{DX}+2 \mathrm{~J}$ motifs, but $\mathbf{B}^{\#}$ contains a $P v u$ II restriction site missing in $\mathbf{D}^{*}$. The expectation is that restriction of $\mathbf{B}^{\#}$ with $P v u I I$ will convert an array with a $32 \mathrm{~nm}$ spacing to one with a $64 \mathrm{~nm}$ spacing. This is indeed what happens (32).

Figure $7 \mathrm{~b}$ illustrates the opposite modification, ligation or annealing to a sticky end. Here, the four DX molecules are labeled $\mathbf{A}$, $\mathbf{B}^{\circ}, \mathbf{C}$ and $\mathbf{D}^{*}$. A, $\mathbf{C}$ and $\mathbf{D}^{*}$ are the same as in Figure $7 \mathrm{a}$, but $\mathbf{B}^{\circ}$ contains a short helix tailed by a sticky end directed out of the plane of the array. The addition of a hairpin tailed by a sticky end complementary to the one on $\mathbf{B}^{\circ}$ should result in the addition of a row of hairpins to the array. This should appear in the AFM as the alteration of an array with a $64 \mathrm{~nm}$ spacing to one with a $32 \mathrm{~nm}$ spacing. Again, this is what is observed (32). This result can be achieved both by covalent ligation and by annealing without ligation, but annealing requires a much longer sticky end.

\section{Periodic Arrays From Holliday-Like Junctions}

The 4-arm junction has been characterized extensively $(33,34)$, because it is an analog (3) of the Holliday (35) recombinational intermediate, containing an asymmetric sequence. We noted above that the solution structure of the Holliday junction entails the stacking of its four arms into two helical domains to form a
Figure 6: Two-Dimensional Patterned Arrays Derived from DX Molecules. (a) A Two Component Array. Two DX molecules are illustrated schematically at the top of this panel, one labeled $\mathbf{A}$ and the other labeled $\mathbf{B}^{*}$. The two helices are represented as rectangles, and the complementary sticky ends are represented by complementary geometrical shapes. The dimensions of each molecule are about $4 \times 16 \mathrm{~nm}$. A is a conventional DX molecule, but $\mathbf{B} *$ is a DX+J molecule, in which a DNA hairpin protrudes from the plane defined by the helix axes of the two antiparallel domains; this hairpin is shown as a filled black circle. Shown below these molecules is an array, drawn half-sized, that shows the two components fitting together to tile a plane. The protruding hairpins of the $\mathbf{B}^{*}$ molecules form rows separated by $32 \mathrm{~nm}$. The topographic features of the DX+J molecules appear as stripes (vertical rows of filled circles) in the AFM, whose resolution is sufficient to resolve stripes, but insufficient to resolve individual hairpins packed together with $4 \mathrm{~nm}$ spacings. (b) A Four Component Array. The same conventions apply as in $(a)$. The difference here is that the array has four components, $\mathbf{A}, \mathbf{B}, \mathbf{C}$ and $\mathbf{D}^{*}$, where $\mathbf{A}, \mathbf{B}$ and $\mathbf{C}$ are conventional DX molecules, and $\mathbf{D}^{*}$ is a $\mathrm{DX}+\mathrm{J}$ motif. It is clear from the bottom portion of the panel that the stripes should be separated by $64 \mathrm{~nm}$, twice the distance seen in $(a)$.

twofold symmetric backbone structure. The axes of these two domains are not coplanar, but are arranged to form a structure twisted by an angle estimated to be about $60^{\circ}$; the estimation has been performed by fluorescence resonance energy transfer (FRET) (36) and by time-resolved FRET (16). Two views of the structure are illustrated in Figures $8 \mathrm{a}$ and $8 \mathrm{~b}$. The view in Figure $8 \mathrm{a}$ is down the twofold axis; in Figure 8b, the structure has been rotated so that the twofold axis is vertical. The view in Figure 8b shows the $60^{\circ}$ angle clearly; Eis and Millar have shown that there is a large dispersion about this angle (16).

One approach that might decrease this dispersion would be to combine several of these molecules together to form a parallelogram-like structure, as illustrated in Figure 8c. The drawing shows that this structure contains two helical domains in one plane, and a second pair in a plane about two nm (a helical thickness) behind it; only the crossovers are coplanar. We have performed this parallelogram by synthesizing and combining the eight strands indicated in Figure 8d. All eight of the arms terminate in sticky ends, A, B, C and D or their complements, $\mathrm{A}^{\prime}$, $\mathrm{B}^{\prime}, \mathrm{C}^{\prime}$ and $\mathrm{D}^{\prime}$. If only two opposite sides contain arms that terminate in sticky ends, say $\mathrm{C}$ and $\mathrm{C}^{\prime}, \mathrm{D}$ and $\mathrm{D}^{\prime}$, it is possible to assemble railroad track-like one dimensional arrays, such as shown in Figure 8e. However, if the molecule shown in Figure $8 \mathrm{~d}$ is used, it is possible to assemble a two dimensional hydrogen bonded array, such as the one shown in Figure 8f, and visualize it by AFM (37).

The basic parallelogram used for these experiments contains six double helical turns in each of its four domains. The crossover sites are located one turn in from the termini, so the large cavity at the center is defined by helices separated by four turns. The repeat distances $(20.5 \pm 0.5$ and $21.1 \pm 0.2 \mathrm{~nm})$ are in good agreement with the expected 6-helical turn repeat $(63 \times 3.4 \AA=$ $21.42 \mathrm{~nm}$ ) for this system (37). We have shown by AFM that one can tune the size of the cavity, by increasing the separation of helices on one pair of edges from four turns to six turns, by using helical domains of lengths six turns and eight turns. The repeat units are now $27.7 \pm 0.8 \mathrm{~nm}$ and $20.9 \pm 0.8 \mathrm{~nm}$, where the longer dimension is in good agreement with a repeat of eight helical turns $(84 \times 3.4 \AA=28.56 \mathrm{~nm})(37)$. 


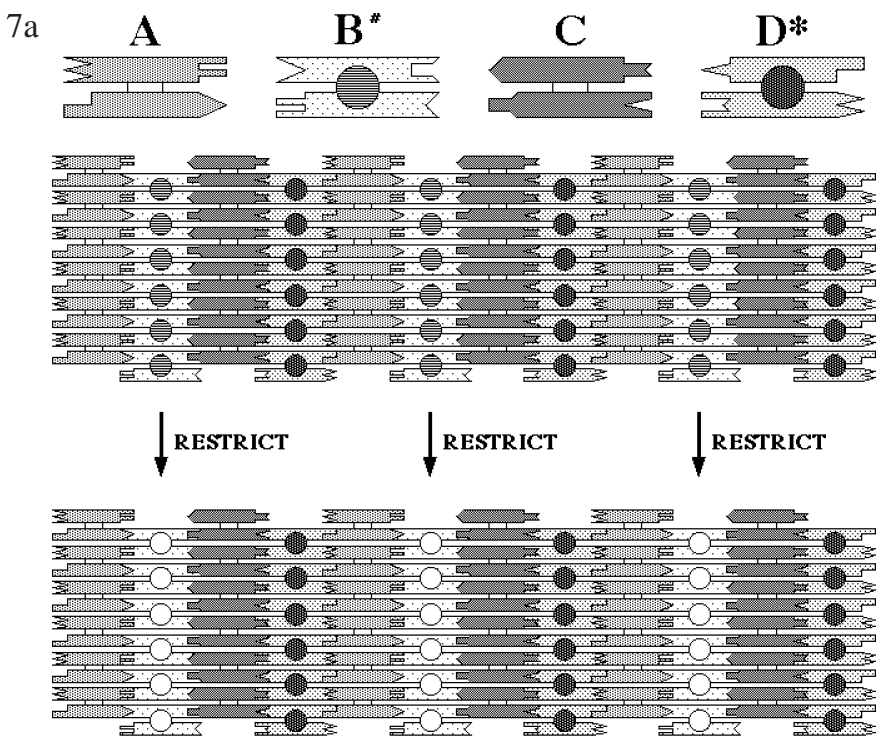

$7 b$
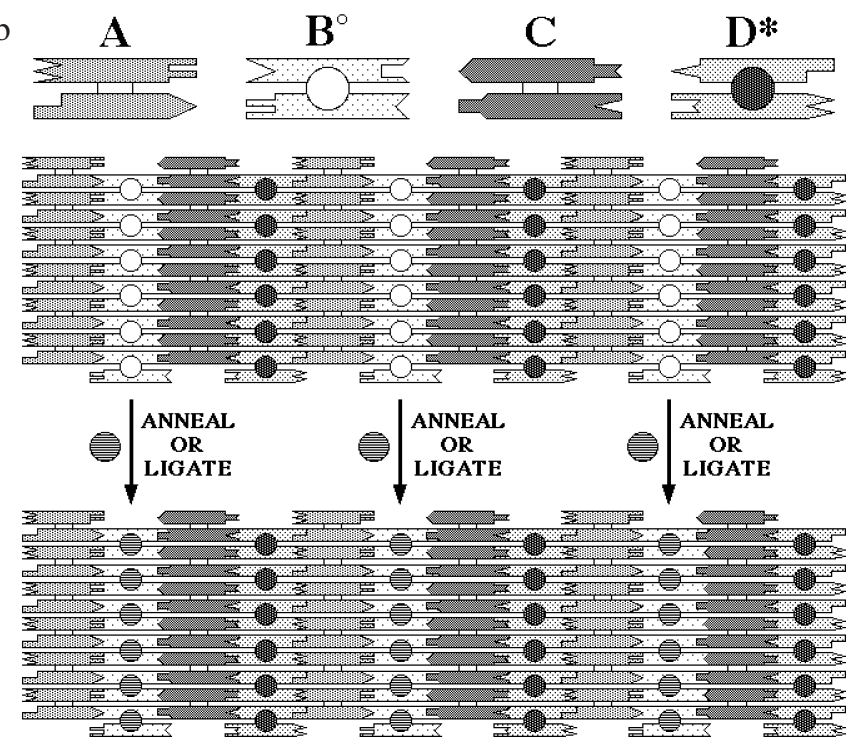

It is of biological interest that one can use this system to measure directly by AFM the angle between the helical domains. So long as the separations are integral multiples of a turn, there are no apparent forces on the angle to prevent it from assuming a value corresponding to the most favorable free energy. This method enables us to average over a large number of unit cells. The angle we observe for the six-by-six turn array is $63.5^{\circ} \pm$ $2.4^{\circ}$, in good agreement with the previous estimates derived from FRET. As a control on the consistency of the measurement, we have also measured the angle in the six-by-eight turn array, which is $62.6 \pm 1.6^{\circ}$ (37).

\section{A DNA Nanomechanical Device}

The sections above describe large arrays whose successful assembly depends critically on the rigidity of their components. There is another application for rigid components, the construction of nanomechanical devices. There are two well-known
Figure 7: The Modification of Two-Dimensional DNA Arrays The same conventions apply as in Figure 6. (a) Restriction of a Crystalline Array. A and $\mathbf{C}$ are DX molecules, and $\mathbf{B}^{\#}$ and $\mathbf{D}^{*}$ are $\mathrm{DX}+2 \mathrm{~J}$ molecules. Their protruding hairpins are represented by filled circles. The different circle fillings indicate that the hairpins differ between the two DX+2J molecules. Directly beneath the components, the array is drawn with the same components reduced in size. The bottom part of the diagram illustrates the effect of removing the hairpin of $\mathbf{B}^{\#}$ by restriction: The prominent stripe is replaced with a much less intense feature. $(b)$ Ligation or Hydrogen Bonded Annealing to a Crystalline Array. The difference between this panel and $(a)$ is that $\mathbf{B}^{\circ}$ replaces $\mathbf{B}^{\#}$. This component contains short arms ending in sticky ends that do not produce an intense feature in the AFM. The drawing illustrates that the addition of hairpins to this array produces a pattern similar to the starting pattern of $(a)$. Annealing and ligation produce the same result, although the sticky ends used for annealing are longer.

transformations of DNA that could be used in principle to produce motion in DNA-based objects. One of these is branch migration, whose dependence on DNA torsion is well known (38). We have used this principle to control the position of the branch point of a partially mobile branched junction, but the requirements for doing this properly lead to an unwieldy and flexible object (39).

A much more fruitful avenue is the use of the B-Z transition (40) to alternate between two well-defined structural states. There are two requirements to form left-handed Z-DNA: An appropriate sequence (called proto-Z DNA) and solution conditions that promote the transition. The sequence requirement allows one to delimit the Z-DNA in space, and the need for proper solution conditions allows one to delimit it in time. Barring direct observation, the most convenient way to observe the two states and their differences is by FRET; selecting two points whose separation should be a direct function of state is a straightforward way to demonstrate motion. We tried unsuccessfully to achieve this goal for nearly a decade, because the components we employed were not rigid. When we discovered that DX molecules were stiff, we decided to incorporate them in a nanomechanical device.

The device and its operation are illustrated in Figure 9. It consists of two DAO molecules connected by a 4.5 turns of double helical DNA. The connecting segment contains a 20-nucleotide length of proto-Z DNA. In the absence of Z-promoting conditions, the proto-Z DNA will be in the B conformation, as shown at the top of Figure 9; however, when the molecule is transferred to Z-promoting conditions it will assume the left-handed ZDNA structure shown at the bottom of Figure 9. The difference in twist for 20 nucleotides converted from B-DNA to Z-DNA corresponds to approximately 3.5 helical turns; it is the half-turn left over that produces the signal indicating motion. The reagent we use to induce the Z-DNA conformation is $\mathrm{Co}\left(\mathrm{NH}_{3}\right)_{6} \mathrm{Cl}_{3}$.

The filled and unfilled circles in Figure 9 represent two fluorescent dyes, fluorescein and Cy3, whose energy transfer is monitored by FRET. The drawing shows that the separation between the two dyes is larger in the Z-state than in the B-state. If one calls the separation $R$, energy transfer is a function of $R^{-6}$; thus, we expect it to decrease when the system is switched from the B-state to the Z-state. This is exactly what happens: Energy 
(a)

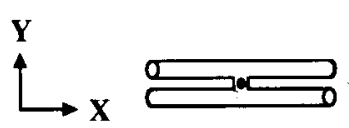

(c)

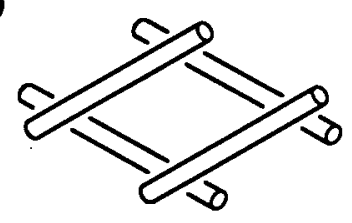

(b)

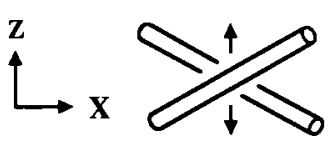

(d)

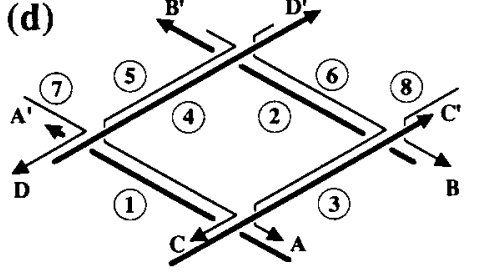

(e)

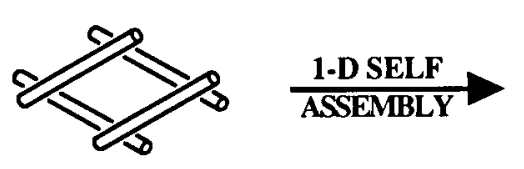

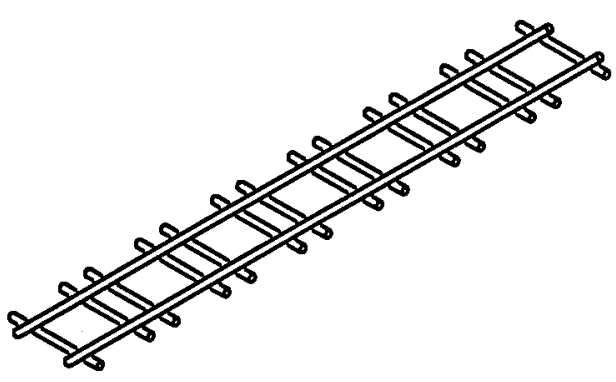

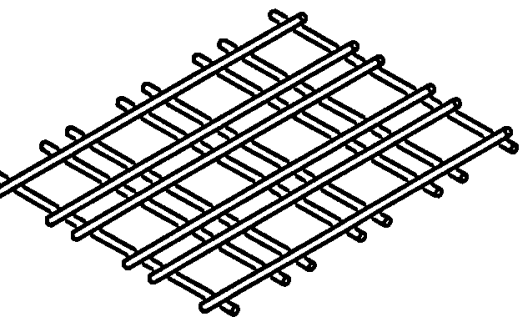

Figure 8: Schematic Representations of Parallelogram-Like Molecular Components and their Assembly. (a)A View Down the Dyad Axis of the Holliday Junction. The dyad axis is indicated by the small lens-shaped figure. The upper helical domain is rotated $30^{\circ}$ about the vertical so that its right end penetrates the page, and the lower helical domain is rotated $30^{\circ}$ about the vertical so that its left end penetrates the page. The $\mathrm{X}$ and $\mathrm{Y}$ axes of a right-handed coordinate system are shown to help orient the reader. (b) $A$ View with the Dyad Axis Vertical. The molecule has been rotated $90^{\circ}$ about the $\mathrm{X}$ axis, as indicated. The dyad axis is indicated by the double arrows. (c) The Combination of Four Junctions into a Parallelogram-Like Motif. Four molecules, in the orientation of (b) are combined. There are six turns of DNA in each helix, and four turns between crossover points, leading to 1-turn overhangs on the ends. Whereas the helical domains are the same length and so are the separations of the crossover points, this particular parallelogram is actually a rhombus. (d) The Strand Structure of the Parallelogram-Like Motif. The strand structure of the molecule in (c) is shown. Strands that are continuous helices (numbered 1-4) are drawn with a thick line, and those involved in crossovers (numbered 5-8) are drawn with a thin line. Arrowheads indicate $3^{\prime}$ ends of strands. Sticky ends are shown by the letters A, B, C and $\mathrm{D}$, and $\mathrm{A}^{\prime}, \mathrm{B}^{\prime}, \mathrm{C}^{\prime}$ and $\mathrm{D}^{\prime}$, respectively, represent their complements. The molecule is constructed from the strands synthesized as shown, rather than from the ligation of four junctions. (e) One-Dimensional Self-Assembly of the Motif into an Array. Onedimensional self-assembly is shown to produce a railroad tracklike arrangement, with helices representing two 'rails'; extending for the length of the assembly and 'ties' separated by alternating distances of four turns and two turns. $(f)$ Two Dimensional SelfAssembly Produces a Latticework of DNA. The array shows the 2-dimensional self-assembly product of the motif; the long separations between helices contain four helical turns, and the short separations contain two helical turns. Note that the latticework array contains two separate layers, an upper layer oriented from lower left to upper right, and a lower layer oriented from lower right to upper left.

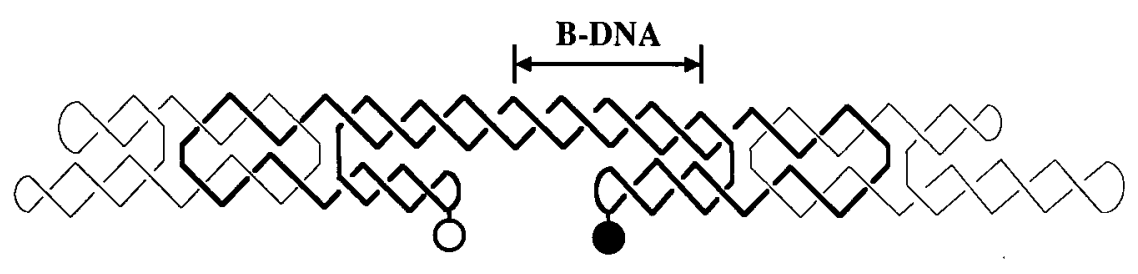

${ }^{2 x} \|^{\mathrm{kz}}$

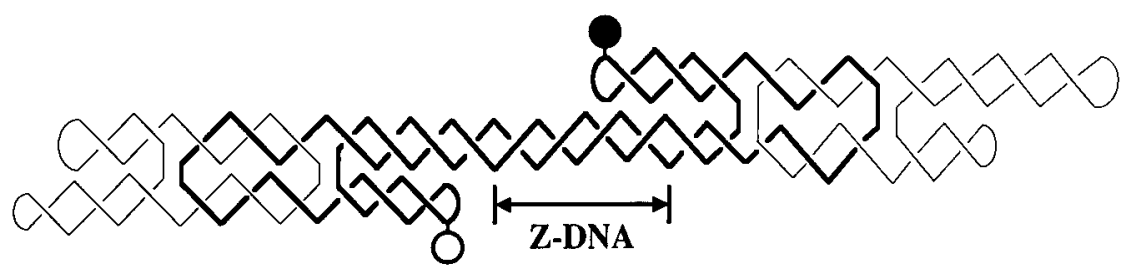

Figure 9: The DNA Nanomechanical Device. The device consists of two DNA DX molecules (DAO motif) connected by 4.5 turns of DNA between the nearest crossover points. The upper portion of the drawing illustrates the molecule constructed entirely from right-handed B-DNA. Three strands are shown, one in the center drawn with a thick line, and two on the ends triply catenated to it. Fluorescent dyes are drawn schematically as filled (fluorescein) and unfilled (Cy3) circles attached to the free hairpins near the middle of the molecule. At the center of the connecting helix is a 20 nucleotide region of proto-Z DNA in the B-DNA conformation. When the B-Z transition takes place, this same portion becomes left-handed Z-DNA, as seen at the bottom of the drawing. When this transition occurs, the two double crossover molecules change their relative positions, increasing the separation of the dyes. It is possible to cycle this system in both directions. 
transfer decreases when the system is put in Z-promoting conditions and it increases when it is removed from those conditions. In a control experiment, a molecule identical in all ways, except lacking the proto-Z segment, shows no condition-dependent change in energy transfer (41).

The motion induced by the $\mathrm{B}-\mathrm{Z}$ transition is expected to be largely rotary. The atoms of the displaced domain nearest the central helix axis are expected to be displaced about $2 \mathrm{~nm}$. However, those furthest from the axis will be displaced by $6 \mathrm{~nm}$. This is a large motion on the scale of existing molecular devices (reference 41 and references therein). In combination with smaller devices, it is possible that one could produce complex, differently-triggered motions that could effect intricate maneuvers on the nanometer scale.

\section{Concluding Remarks}

The results reported here are all dependent on the exploitation of rigid DNA motifs, DX molecules and parallelograms made from Holliday junction analogs. These components have allowed us to take a major step in the design of matter on the mesoscopic scale. We are able to design molecular components whose structural features result in specific and programmable patterns; these patterns can be visualized in two dimensions by AFM. The DX molecules contain protrusions that merge into patterns, and the parallelograms contain cavities whose dimensions are tunable. In addition to forming specific patterns with the DX molecules, we can modify the patterns in an economical fashion: given a well-defined starting point and orientation, ten differently composed stripes could result in over a thousand different patterns (32). The use of simple starting components has enabled the convenient assembly of micron-sized arrays, and augurs well for much larger species. In addition to these results, we have demonstrated that it is possible to produce a nanomechanical device from DNA.

What goals remain to be achieved in these directions? The goal of assembling three-dimensional periodic matter, first enunciated at the Second Conversation in Biomolecular Stereodynamics (42), remains elusive. This aim is key, not only because it may contribute to solving the problem of macromolecular crystallization $(3,42)$ and to the assembly of molecular electronic components (22). On a more fundamental level the rationally-designed self-assembly of crystalline matter on the macroscopic scale will form a link between the microscopic and the macroscopic that will enable us to design new materials with unprecedented properties. This target will be particularly valuable if we can incorporate heteromolecules into DNA arrays, so that their properties and interactions can be exploited. Finally, the incorporation of nanomechanical devices, such as the one described here, into those systems holds the promise of very smart materials, indeed.

\section{Acknowledgments}

This work has been supported by grants GM-29554 from the National Institute of General Medical Sciences, N00014-89-J-
3078 from the Office of Naval Research, NSF-CCR-97-25021 from DARPA/National Science Foundation and F30602-98-C0148 from the Information Directorate of the Rome NY Air Force Research Laboratory to N.C.S., by Margaret and Herman Sokol Fellowships to J.C. and X.Y., and by NYU-GSAS Dissertation Fellowships to Y.Z. and C.M.

\section{References and Footnotes}

1. Watson, J.D. \& Crick, F.H., Nature (London) 171, 737-738 (1953).

2. Caruthers, M.H., Science 230, 281-285 (1985).

3. Seeman, N.C., J. Theor. Biol. 99, 237-247 (1982).

4. Seeman, N.C., Ann. Rev. Biophys. Biomol. Str. 27, 225-248 (1998).

5. Seeman, N.C., Angew. Chemie Intl. Ed. 37, 3220-3238 (1998).

6. Adleman, L.M., Science 266, 1021-1024 (1994).

7. Winfree, E., In: DNA Based Computers, ed. by R. Lipton and E. Baum, Am. Math. Soc., Providence (1995), pp. 199-215.

8. Cohen, S.N., Chang, A.C.Y., Boyer, H.W. \& Helling, R.B., Proc. Nat. Acad. Sci. (USA) 70, 3240-3244 (1973).

9. Wells, A. F., Three-dimensional Nets and Polyhedra, John Wiley \& Sons, New York (1977).

10. Williams, R., The Geometrical Foundation of Natural Structure, Dover, New York(1979).

11. Ma, R.-I., Kallenbach, N.R., Sheardy, R.D., Petrillo, M.L. \& Seeman, N.C., Nucl. Acids Res. 14, $9745-9753$ (1986).

12. Kallenbach, N.R., Ma, R.-I. \& Seeman, N.C., Nature 305, 829-831 (1983).

13. Wang, Y., Mueller, J.E., Kemper, B., \& Seeman, N.C., Biochem 30, 56675674 (1991). 
40. Rich, A., Nordheim, A. \& Wang, A.H.-J., Ann. Rev. Biochem. 53, 791-846 (1984).

41. Mao, C., Sun, W., Shen, Z. \& Seeman, N.C., Nature 397, 144-146 (1999).

42. Seeman, N.C., In: Biomolecular Stereodynamics, ed. by R.H. Sarma, Adenine Press, New York (1981), pp. 269-277. 